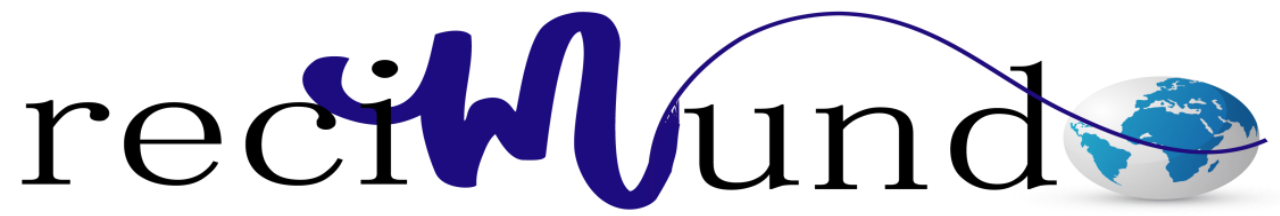

Revista Científica Mundo de la Investigación y el Conocimiento

Karina Jessenia Jarrin Maisincho a ; William Plúa Marcillo b; José Javier Asencio Mejia $^{\text {c. }}$ Nicole Sánchez Figueroa ${ }^{\mathrm{d}}$

Manejo endoscópico de fístula gástrica posterior a gastrectomía vertical

Revista Científica Mundo de la Investigación y el Conocimiento. Vol. 2 núm., 1, febrero, ISSN: 2588-073X, 2018, pp. 190-201

DOI: 10.26820/recimundo/2.1.2018.190-201

Editorial Saberes del Conocimiento

Recibido: 05/12/2017

Aceptado: 10/02/2018

a. Profesional Independiente; drakarinajarrinmcd@hotmail.com

b. Cirujano Bariátrico, Servicio de Cirugía General - Hospital Teodoro Maldonado Carbo, Guayaquil - Ecuador

c. Médico Posgradista de Cirugía General de la Universidad de Especialidades Espíritu Santo - Hospital Teodoro Maldonado Carbo, Guayaquil - Ecuador

d. Médico, Clínica Grifi's, Guayaquil - Ecuador 
Manejo endoscópico de fístula gástrica posterior a gastrectomía vertical

Vol. 2, núm. 1., (2018)

Karina Jessenia Jarrin Maisincho; William Plúa Marcillo; José Javier Asencio Mejia; Nicole

Sánchez Figueroa

\section{RESUMEN}

Las fístulas gástricas se reportan como una de las complicaciones en pacientes sometidos a gastrectomía vertical laparoscópica. A pesar de ser poco frecuentes, representan un alto índice de mortalidad debido a su infra-diagnóstico. El manejo endoscópico por medio de endo-prótesis supone una de las alternativas como tratamiento conservador reduciendo considerablemente la morbimortalidad posterior a una re-intervención quirúrgica. Se presenta un caso de fístula gástrica tardía que ingresa a urgencias con datos clínicos y bioquímicos de shock séptico. El caso se estudió con tomografía computarizada de tórax, tránsito gastro-intestinal y finalmente se confirmó con endoscopía evidenciando fístula gástrica a nivel de antro. La paciente recibió tratamiento conservador endo-prótesis. Luego de 6 semanas se retira la prótesis evidenciándose cierre completo de la fístula.

Palabras clave: Gastrectomía; cirugía bariátrica; fuga anastomótica; fístula gástrica; laparoscopía; endoprótesis. 


\title{
Manejo endoscópico de fístula gástrica posterior a gastrectomía vertical
}

Vol. 2, núm. 1., (2018)

Karina Jessenia Jarrin Maisincho; William Plúa Marcillo; José Javier Asencio Mejia; Nicole Sánchez Figueroa

\begin{abstract}
Gastric fistulas are reported as one of the complications in patients undergoing laparoscopic vertical gastrectomy. Although rare, they represent a high mortality rate due to their underdiagnosis. Endoscopic management with usage of endo-prosthesis is one of the alternatives as a conservative treatment, considerably reducing morbidity and mortality following surgical reintervention. A case of a patient with late gastric fistula who is admitted to the emergency department with clinical and biochemical data of septic shock is presented. The case was studied with computed tomography of the chest, gastro-intestinal transit and finally confirmed with endoscopy, which evidences a gastric fistula at the antrum. The patient received conservative endo-prosthesis treatment. After 6 weeks the prosthesis is removed, showing complete closure of the fistula.
\end{abstract}

Keywords: Gastrectomy; bariatric surgery; obesity; anastomotic leak; gastric fistula; laparoscopy; endoprosthesis 


\section{Manejo endoscópico de fístula gástrica posterior a gastrectomía vertical}

Vol. 2, núm. 1., (2018)

Karina Jessenia Jarrin Maisincho; William Plúa Marcillo; José Javier Asencio Mejia; Nicole

Sánchez Figueroa

\section{Introducción.}

La cirugía bariátrica reduce el riesgo relativo de muerte en un $89 \%$ porque permite la pérdida de peso, corrigiendo factores de riesgo cardiovasculares y disminuyendo el riesgo de cáncer (1). La gastrectomía vertical laparoscópica (GVL) es un abordaje quirúrgico para tratar la obesidad mórbida. Ésta restringe el tamaño del estómago para inducir la saciedad y reseca las células productoras de grelina del fundus para disminuir el apetito (2). Ésta está asociado a $>50 \%$ de pérdida del exceso de peso en 5 años (3). GLV se ha convertido en un procedimiento muy frecuente en cirugía bariátrica debido a su simplicidad y eficacia comparado con el procedimiento de derivación o bypass (4). Sin embargo, comparado con el bypass gástrico o desviación biliopancreática, sus complicaciones pueden ser incluso más severas (5). Las fugas por la línea de grapado, el sangrado y las estenosis son las complicaciones comúnmente reportadas después de GVL. Según la información obtenida de 12799 GVL por el International Sleeve Gastrectomy Panel Consensus Statement en el 2011, la tasa de fugas es del 1.06\% (6), pero puede variar entre el $1 \%$ y el $3 \%$ en un procedimiento primario y más del $10 \%$ en procedimientos secundarios (2). La fístula gástrica es la complicación más seria posterior a la GVL con una incidencia que varía del 0 al 5\%, de acuerdo a Rebibo et al (7).

De acuerdo al Royal College of Surgeons of England, una fuga gástrica se define como el escape de contenido luminal desde una unión quirúrgica entre dos vísceras huecas. También puede ser una efluente de contenido gastrointestinal a través de una línea de sutura, que puede acumularse cerca de la anastomosis o salir a través de la pared o el dren (8). Una fuga gástrica puede convertirse en una fístula, con lo que clínicamente se manifiesta como salida de líquido gástrico a través de los drenes o de la pared abdominal (9) (10). 


\section{Manejo endoscópico de fístula gástrica posterior a gastrectomía vertical}

Vol. 2, núm. 1., (2018)

Karina Jessenia Jarrin Maisincho; William Plúa Marcillo; José Javier Asencio Mejia; Nicole Sánchez Figueroa

Las fugas pueden clasificarse en relación al tiempo inicio, presentación clínica, apariencia radiográfica o una mezcla de factores (2). Csendes et al definió a las fugas como tempranas, intermedias y tardías a aquellas que aparecen 1 a 4 días, 5 a 9 días y 10 o más días posterior a la cirugía, respectivamente. Por severidad de las manifestaciones clínicas y extensión de la diseminación, ellos definieron la fuga tipo I o subclínica a aquellas que están bien localizadas sin diseminación a la cavidad pleural o abdominal, no hay manifestaciones clínicas sistémicas, son fáciles de tratar médicamente. Las fugas tipo II son aquellas con diseminación al espacio pleural o cavidad abdominal o a los drenes con consecuentes manifestaciones sistémicas severas (11) (12). Basado en hallazgos tanto clínicos como radiológicos, las fugas tipo A son micro-perforaciones sin evidencia clínica o radiológica de fuga. Mientras que las fugas tipo B son detectadas por estudios radiológicos pero sin presentar clínica. Finalmente las fugas tipo C presentan evidencia clínica y radiológica (13). Por lo tanto las fugas tipo II y tipo C se incluyen dentro de la definición de fístulas gástricas.

Las fugas gástricas pueden darse por causas mecánicas o isquémicas (2). En un estudio multi-céntrico con 2834 pacientes, las fugas post GLV incluyeron: vascularización inadecuada debido a una disección agresiva especialmente de las uniones posteriores de la manga superior, lesiones térmicas en el tubo gástrico por dispositivos ultrasónicos (harmonic, Ligasure) y fallas en los dispositivos de engrapado, engrapado en el tubo oro-gástrico (14).

La presentación clínica puede variar desde aquel paciente totalmente asintomático diagnosticados con estudios de imagen de rutina, considerados tipo A como ya se mencionó anteriormente, hasta aquel paciente con signos y síntomas de shock séptico incluyendo fiebre, dolor abdominal, peritonitis, leucocitosis, taquicardia, hipotensión (13). Estudios de laboratorio 


\section{Manejo endoscópico de fístula gástrica posterior a gastrectomía vertical}

Vol. 2, núm. 1., (2018)

Karina Jessenia Jarrin Maisincho; William Plúa Marcillo; José Javier Asencio Mejia; Nicole

Sánchez Figueroa

incluyendo biometría hemática completa, PCR, no son ni sensibles ni específicas y raramente contribuyen a realizar un diagnóstico definitivo (14). Sin embargo se recomienda no dar de alta a ningún paciente con presencia de signos o síntomas de shock como taquicardia, escalofríos o fiebre, así como elevación de PCR o presencia de azul de metileno en los drenajes, ya que se debe sospechar de una fístula gástrica temprana (15).

Basado en el First International Summit for Sleeve Gastrectomy, el tratamiento puede incluir re-suturar, drenaje (abierto o laparoscópico), clipaje endoscópico, colocación de stents o endo-prótesis, uso de pegamento de fibrina, a veces el uso de una Y de Roux o gastrectomía total como último recurso (9) (10) (11). Las fugas gástricas generalmente tardan más de 6 semanas en cicatrizar (promedio de 45 días), en comparación con el tiempo de cicatrización en las derivaciones de bypass gástrico laparoscópico Roux en Y (30 días) (11). Por otro lado varios autores concuerdan en que 6 a 8 semanas es el tiempo óptimo para la extracción, ya que un periodo más prolongado incrementa el riesgo de dañar la mucosa subyacente especialmente con los stents descubiertos (14) (16).

\section{Reporte de caso}

Paciente femenino de 29 años con diagnósticos de hipertensión arterial, intolerancia a la glucosa y obesidad mórbida (IMC de 41.3) se le realizó GVL sin incidencias y con evolución postoperatoria satisfactoria. Un mes posterior a la intervención quirúrgica ingresa a urgencias por fiebre $\left(39^{\circ} \mathrm{C}\right)$, astenia, hiporexia, tos productiva y descompensación hemodinámica. Exámenes de laboratorio revelan leucocitosis y aumento de reactantes de fase aguda. Estudios de imágenes: TC de tórax refleja proceso condensativo en segmento basal postero-medial de ambos lóbulos 


\section{Manejo endoscópico de fístula gástrica posterior a gastrectomía vertical}

Vol. 2, núm. 1., (2018)

Karina Jessenia Jarrin Maisincho; William Plúa Marcillo; José Javier Asencio Mejia; Nicole Sánchez Figueroa

inferiores. TC abdomino-pélvica S/C revela presencia de colección sub-frénica izquierda (80x50 $\mathrm{mm}$ ) con aire en su interior adyacente a la línea de grapado del estómago. Paciente es derivada a UCI para manejo de shock séptico. Se realizó laparoscopía quirúrgica más drenaje de colección intra-abdominal y se evidenció fístula gástrica a nivel de antro. El tránsito EGI evidencia extravasación del contraste y la endoscopía reveló orificio fistuloso $(20 \mathrm{~mm})$ en cara posterior a 3 cm del cardias.
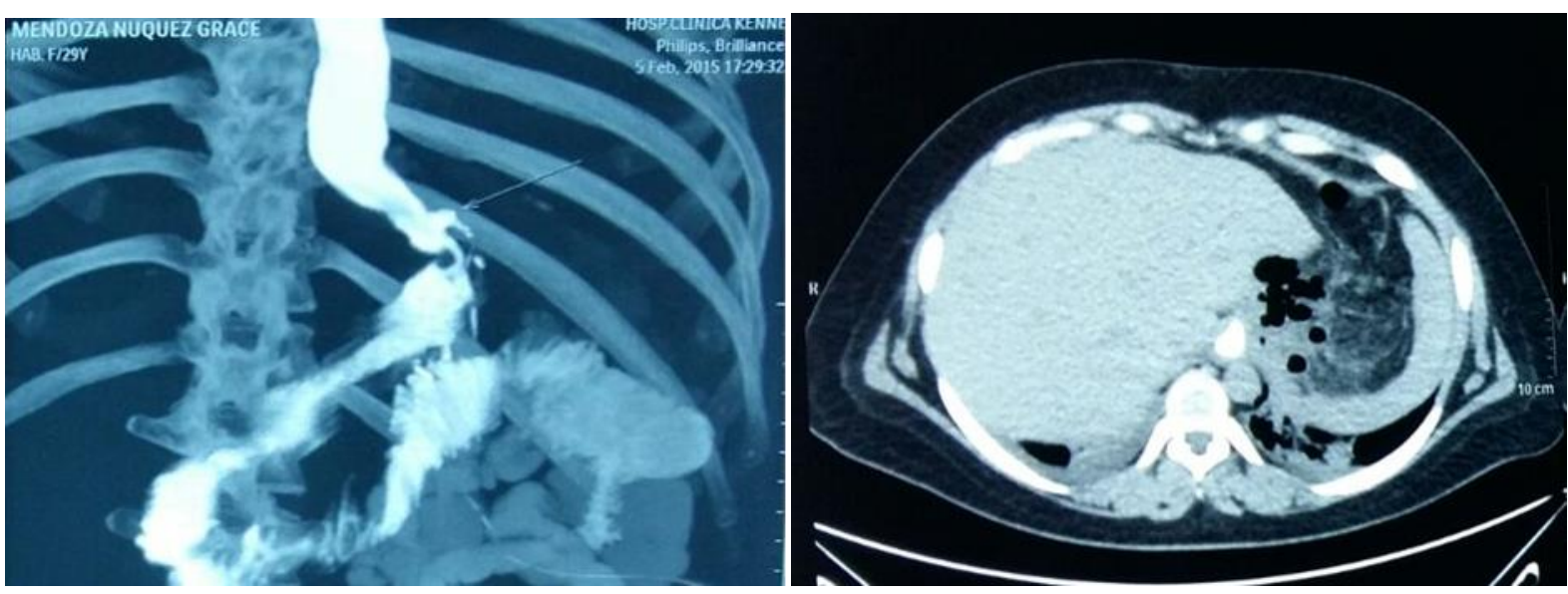

Fig 1. TAC con contraste oral donde se evidencia fistula gástrica, y presencia de aire adyacente a línea de grapado.

Se decide manejo endoscópico de la fístula colocando endo-prótesis gástrica la cual en 72 horas migró y fue sustituida por una de mayor tamaño $(103 \times 18 \mathrm{~mm})$. La prótesis se retiró a las 6 semanas demostrándose el cierre completo de la fístula con buena respuesta al manejo médico. 
Manejo endoscópico de fístula gástrica posterior a gastrectomía vertical

Vol. 2, núm. 1., (2018)

Karina Jessenia Jarrin Maisincho; William Plúa Marcillo; José Javier Asencio Mejia; Nicole

Sánchez Figueroa

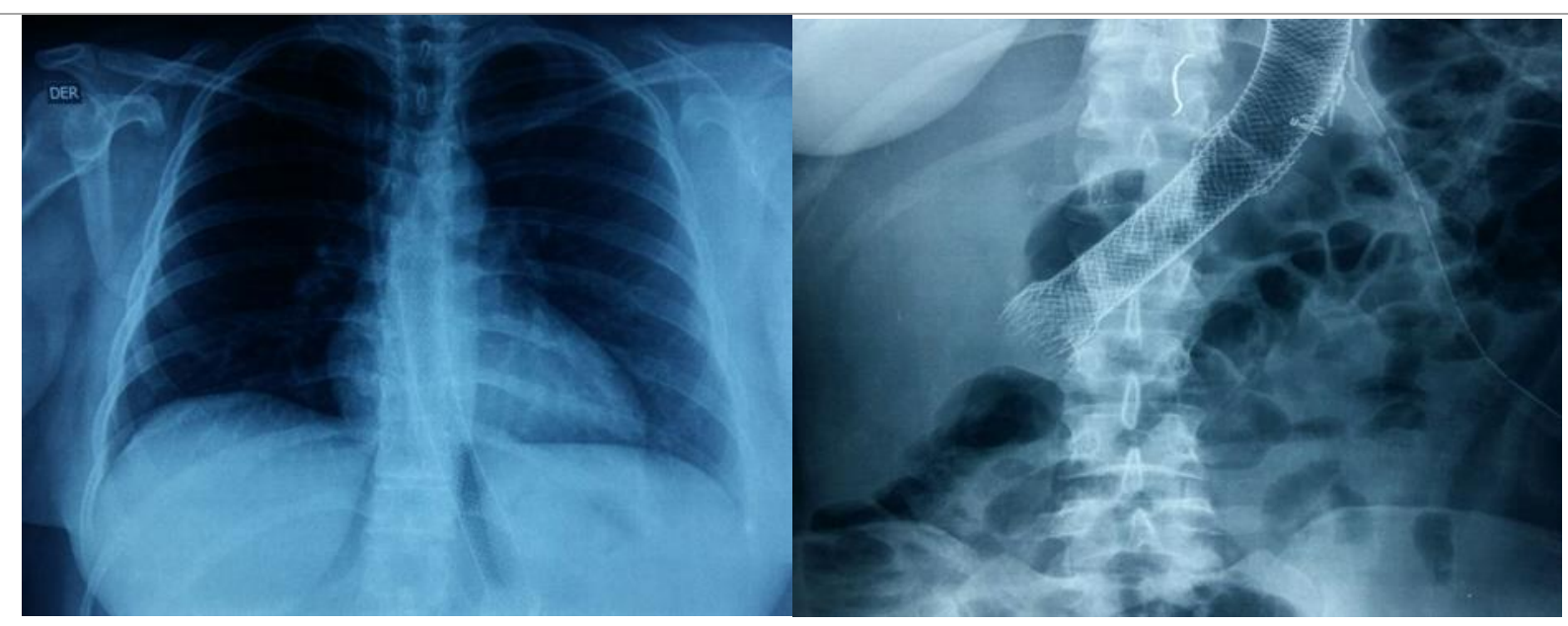

Fig 2. Rx. Torax y Abdomen donde se observa la posición del stent

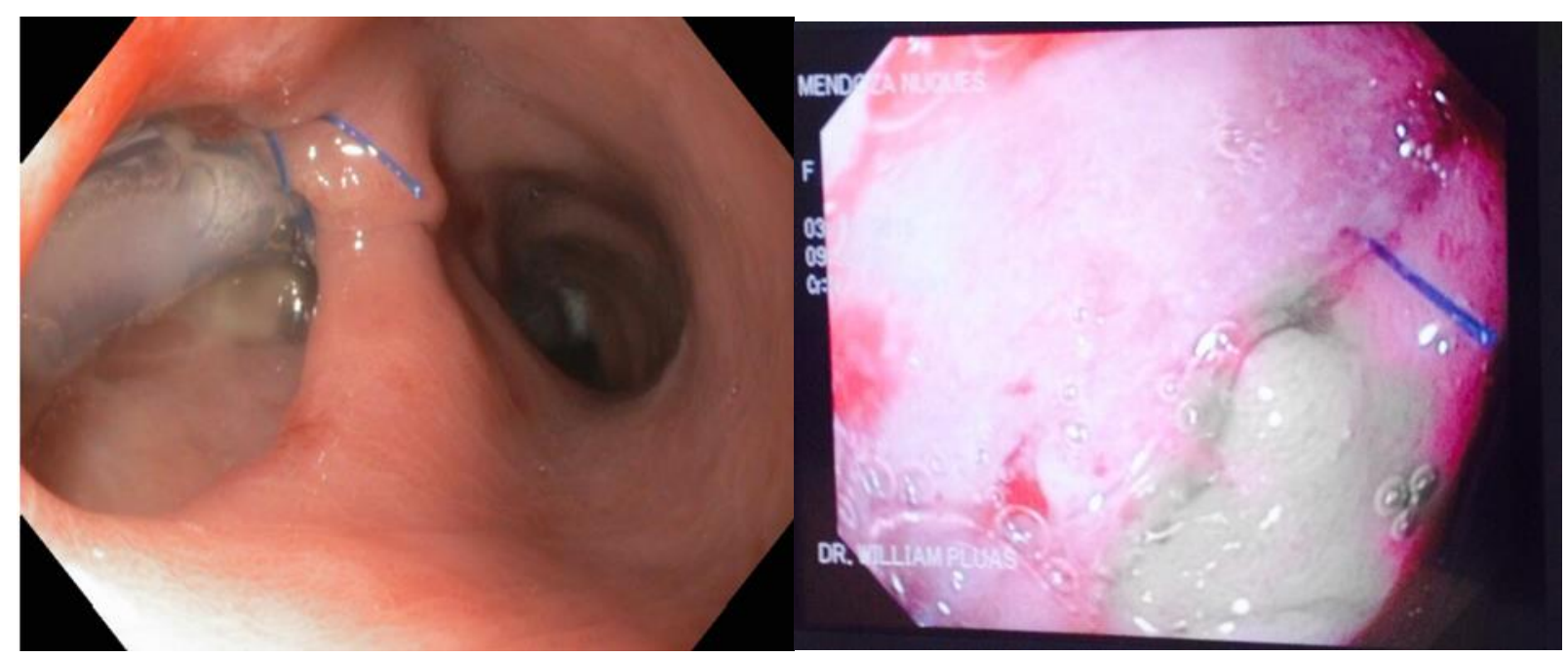

Fig. 3 Endoscopia Digestiva Alta A, revela orificio fistuloso gástrico de $20 \mathrm{~mm}$. B, resolución de fistula gástrica

\section{Discusión.}

El tipo de presentación del presente caso es similar al que reporta la literatura. Praveenraj et al describió que los pacientes de su estudio con fuga gástrica presentaron dolor abdominal, 


\section{Manejo endoscópico de fístula gástrica posterior a gastrectomía vertical}

Vol. 2, núm. 1., (2018)

Karina Jessenia Jarrin Maisincho; William Plúa Marcillo; José Javier Asencio Mejia; Nicole Sánchez Figueroa

fiebre, taquicardia, tos y neumonía y que fue diagnosticada en tiempos que varían de 24 horas a 7 meses después de la cirugía. Al igual que el presente caso, los pacientes de su estudio que presentaron fuga gástrica tardía se encontraban bien al inicio del post operatorio y después del alta presentaron fiebre, dolor abdominal, tos y/o sepsis y también en ellos se encontraron colecciones subdiafragmáticas. La fuga gástrica se ubicó en el tercio proximal de la curvatura mayor en toda su muestra (12).

Si durante el transoperatorio se sospecha de compromiso de la línea de grapeo, se puede utilizar la prueba de azul de metileno para observar el sitio probable de fuga (10). Sin embargo, puede teñir tejidos y estructuras adyacentes dificultando la identificación y la re-sutura (12). Otro estudio que puede ser usado durante el transoperatorio es la gastroscopia, que permite identificar y reparar al instante la fuga. Ninguno de los anteriores previene aparición de fuga gástrica tardía (10). Si existe alta sospecha clínica de fuga gástrica en el post operatorio debe realizarse tomografía computarizada con contraste la cual diferenciará las fugas localizadas de la diseminación difusa e identificará abscesos o tractos fistulosos (12). Sin embargo, la sensibilidad de ésta disminuye con el grado de obesidad, por lo cual no es útil en pacientes con IMC >50 (10).

No se han realizado estudios de prevalencia en el Ecuador, pero el porcentaje de complicaciones de cirugía bariátrica se encuentra entre 4 al 22\% (16). Dentro de los tratamientos quirúrgicos de la obesidad mórbida, la GVL es uno de los procedimientos que se realiza en nuestro hospital. El tratamiento de fístula gástrica requiere cuidado intensivo e involucra cirujanos, radiólogos y endoscopistas (17). En un $80 \%$ de los casos de fístulas gástricas se requiere cirugía de revisión y un promedio de cinco procedimientos endoscópicos (18). En 


\section{Manejo endoscópico de fístula gástrica posterior a gastrectomía vertical}

Vol. 2, núm. 1., (2018)

Karina Jessenia Jarrin Maisincho; William Plúa Marcillo; José Javier Asencio Mejia; Nicole

Sánchez Figueroa

pacientes con inestabilidad hemodinámica se debe realizar un lavado de cavidad abdominal de inmediato y colocación de drenajes para luego una reintervención quirúrgica más invasiva como Y de Roux o gastrectomía total (11) (17). Si es una fuga tardía con estabilidad hemodinámica, el manejo conservador puede realizarse con antibiótico terapia de amplio espectro, drenaje percutáneo, inhibidores de la bomba de protones a altas dosis (9) y el aspecto nutricional es muy importante ya que de ellos depende la cicatrización de los tejidos posterior a la fuga (10). El tratamiento conservador de elección en nuestro paciente fue el manejo endoscópico mediante endo-prótesis el cual llevó a la completa resolución de la fistula gástrica, reduciendo así el porcentaje de morbimortalidad de la paciente. Ésta es una técnica de mínimo acceso con baja tasa de complicaciones evitando así procedimientos invasivos (10).

\section{Conclusiones.}

La fuga gástrica de la paciente del caso clínico expuesto corresponde a fuga tipo C, fístula gástrica con manifestaciones clínicas y evidencia radiológica. El manejo conservador de fistula gástrica tardía mediante endoscopía con endo-prótesis demostró un resultado eficaz al ser retirado a la semana 6 . La sospecha clínica y el uso de tomografía computarizada con contraste constituyen el pilar diagnóstico de fugas post quirúrgicas. El tiempo de cierre de la fuga puede ser más corto con la intervención quirúrgica y/o endoscopía que el manejo expectante. El manejo, endoscópico o reintervención quirúgica debe individualizarse según la presentación clínica. Frecuentemente se requiere una combinación de métodos según la situación para lograr el éxito. 


\section{Manejo endoscópico de fístula gástrica posterior a gastrectomía vertical}

Vol. 2, núm. 1., (2018)

Karina Jessenia Jarrin Maisincho; William Plúa Marcillo; José Javier Asencio Mejia; Nicole Sánchez Figueroa

\section{Bibliografía.}

1. Sjostrom L. Bariatric surgery and reduction in morbidity and mortality: experiences from the SOS study. International Journal of Obesity. 2008; 32(7): p. 93-97.

2. Abou AR, Basile M, El Masri H. Gastric leaks post sleeve gastrectomy: Review of its prevention and management. World Journal of Gastroenterology. 2014 october 14; 20(38): p. 13904-10.

3. Kehagias I, Spyropoulos C, karamanakos S. Efficacy of sleeve gastrectomy as sole procedure in patients with clinically severe obesity (BMI $\leq 50 \mathrm{~kg} / \mathrm{m}(2))$. Surgery for Obesity and Related Diseases. 2013 junio; 9(3): p. 363-9.

4. Msika S, Castel B. Present indications for surgical treatment of morbid obesity: how to choose the best operation? Journal of Visceral Surgery. 2010 october; 147(5): p. e47-e51.

5. Ferrer Marquez M, Ferrer Ayza M, Belda Lozano R. Gastric Leak After Laparoscopic Sleeve Gastrectomy. Obesity Surgery. 2010 september; 20(9): p. 1306-1311.

6. Rosenthal R. Surgery for Obesity and Related Diseases. 2012; 8(1): p. 8-19.

7. Rebibo L, Fuks D, Blot C. Gastrointestinal bleeding complication of gastric fistula after sleeve gastrectomy: consider pseudoaneurysms. Surgical Endoscopy. 2013 february 8; 27(8): p. $2849=2855$.

8. Peel A, Taylor E. Proposed definitions for the audit of postoperative infection: a discussion paper. Surgical Infection Study Group. Annals Royal College of Surgeons of England. 1991 november; 73(6): p. 385-388.

9. Bekheit M, Katri K, Nabil W. Earliest signs and management of leakage after bariatric surgeries: Single institute experience. Alexandria Journal of Medicine. 2013 march; 49(1): p. 29-33.

10. Márquez-González SM, Ceró. Fuga gástrica posterior a gastrectomía en manga laparoscópica. Clasificación, diagnóstico y tratamiento. CIRUGÍA ENDOSCÓPICA. 2016 oct-dic; 17(4): p. 191-5.

11. Csendes A, Braghetto I, Leon P, Burgos AM. Management of Leaks After Laparoscopic Sleeve Gastrectomy in Patients with Obesity. Journal of Gastrointersinal Surgery. 2010 


\section{Manejo endoscópico de fístula gástrica posterior a gastrectomía vertical}

Vol. 2, núm. 1., (2018)

Karina Jessenia Jarrin Maisincho; William Plúa Marcillo; José Javier Asencio Mejia; Nicole

Sánchez Figueroa

september; 14(9): p. 1343-8.

12. Praveenraj P, Gomes, Kumar, Parthasarathi. Management of gastric leaks after laparoscopic sleeve gastrectomy for morbid obesity: A tertiary care experience and design of a management algorithm. Journal of Minimal Access Surgert. 2016; 12(4): p. 342-9.

13. Welsch T, Von Frankenberg M. Diagnosis and definition of anastomotic leakage from the surgeon's perspective. 2011 enero; 82(1): p. 48-55.

14. Sakran N, Goitein D, Raziel A, Keidar A. Gastric leaks after sleeve gastrectomy: a multicenter experience with 2,834 patients. Surgical Endoscopy. 2013 january; 27(1): p. 2405.

15. Gagnière , Slim. Don't Let Obese Patients Be Discharged with Tachycardia After Sleeve Gastrectomy. Obesity Surgery. 2012 september ; 22(9): p. 1519-1520.

16. Alastrué , Botey M, Moreno P, Balibrea J. Manejo novedoso de las fugas del tubo gástrico Revisión de la Literatura. Bariatrica \& metabolica Ibero-Americana. 2012 diciembre; 2(3): p. 93-100.

17. Aurora A, Khaitan L, Saber A. Sleeve gastrectomy and the risk of leak: a systematic analysis of 4,888 patients. Surgical Endoscopy. 2012 junio; 26(6): p. 1509-15.

18. Peguinot A, Fuks A. Is there a place for pigtail drains in the management of gastric leaks after laparoscopic sleeve gastrectomy? Obesity Surgery. 2012 may; 22(5): p. 712-20. 